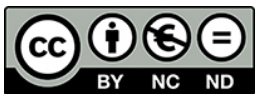

\title{
De la réalité des modèles et des théories
}

\author{
François Anceau (Author) \\ Professeur CNAM émérite \\ Paris, France \\ francois.anceau@waika9.com \\ Laurent Carrive (Author) \\ Docteur en Psychopathologie, Ingénieur, DEA de Logique mathématique et de Philosophie \\ Chargé de cours de Philosophie : Université Paris 7 \\ Paris, France \\ 1.carrive@free.fr
}

\begin{abstract}
La réalité des modèles comme des théories fait aujourd'hui l'objet de nombreux débats. Pourtant, ce critère essentiel reste actuellement négligé du fait de l'expansion d'un certain constructivisme, qui admet implicitement une forme d'équivalence entre justesse des prédictions et réalité physique. Dans cet article, nous entendons par modèle physique, un système mathématico-logique prédictif, permettant de simuler le comportement d'un objet. Une théorie, plus générale qu'un modèle, désignera un objet cognitif qui prétend décrire et expliquer la nature et le comportement réel d'un phénomène. Nous considérons ensuite qu'un modèle est opérationnel lorsqu'il décrit précisément les processus physiques qui engendrent l'action. Tandis qu'un modèle sera dit nonopérationnel s'il met en œuvre des évaluations qui ne peuvent être obtenues que rétrospectivement, par exemple par un calcul d'extremum. Enfin, nous qualifions un modèle de réel, s'il est opérationnel et si, de plus, ses variables et opérations sont prises strictement dans le domaine de réalité considéré (en référence aux poupées russes de Bitbol [9]). Une théorie est donc nécessairement un modèle réel, ou supposé tel.
\end{abstract}

Il nous est apparu que les théories physiques sont la plupart du temps, organisées de manière hiérarchique, comportant à leur base des modèles réels surmontés de modèles non-réels. Les modèles réels permettent de découvrir de nouveaux phénomènes, par exemple la découverte de la circulation aérodynamique autour d'une aile d'avion par Nikolaï Joukovski. Par contre, la non-réalité de certains modèles peut conduire à des raisonnements erronés, en suggérant une fausse réalité. Toutefois, les modèles non-réels peuvent être très utiles lorsque les objets manipulés sont inobservables, car trop petits ou imaginaires. La qualité de leurs prédictions peut être aussi bonne que celle fournie par les modèles réels. C'est le cas de la mécanique quantique, développée au début du XXème siècle, qui a montré sa très grande utilité pratique et la surprenante exactitude de ses prédictions. Lors de son développement, les niveaux de réalité concernés étaient alors complètement inobservables. Ils ont donc été imaginés. L'évolution actuelle de la technologie, commence à permettre une certaine visibilité des atomes et des couches électroniques, ce qui pourrait profondément remettre en cause ce modèle en exhibant sa non-réalité.

Keywords: Modèle, théorie, réalité, Physique, Philosophie, Kant, réalité pratique, Mécanique quantique, Réalisme, Idéalisme, macroscopique, microscopique.

\section{INTRODUCTION}

\section{A. Propos}

Avant d'aborder la question difficile de la réalité des modèles scientifiques, nous allons tenter, dans cet exposé, de circonscrire la notion générale de réalité. Les notions de réalité sont nombreuses, complexes et renvoient à une grande diversité de positions allant de l'idéalisme le plus radical aux différentes formes de réalisme. Par ailleurs, les conceptions scientifiques et philosophiques 
de la réalité, du réel, entretiennent entre elles des relations étroites qui prêtent souvent à confusion. L'idéalisme philosophique en particulier, lorsqu'il est mal compris, peut donner lieu à des interprétations scientifiques aberrantes.

C'est pourquoi il nous a semblé pertinent de reconsidérer la notion de «réalité pratique » dans le champ spécifique de la physique et de ses modèles. Celle-ci pourrait constituer un critère raisonnable de réalité, d'une part compatible avec les principales critiques philosophiques restées d'actualité, d'autre part qui rende compte des niveaux de réalité dont traite les modèles physiques et de l'usage dont ils sont issus. Ce critère "pratique », remanié, devrait permettre, d'asseoir plus solidement notre jugement sur l'adéquation des modèles physiques à la réalité et donc de mieux distinguer les théories dites réelles, des théories manquant de réalité.

\section{B. L'importance du rapport Philosophie/Physique}

La science et la philosophie sont nées simultanément chez les Grecs, au VIe siècle av. JC. Longtemps inséparables, elles ont toutes deux poursuivi un but semblable : la recherche de la vérité. Pour étudier la réalité des modèles scientifiques, il est donc essentiel de bien comprendre l'apport de la philosophie, qui est depuis son origine antique, partie prenante de la science et qui l'a même érigée en idéal.

On admet généralement, qu'au XVIIe siècle eut lieu entre elles deux, une forme de rupture, dont Galilée, Descartes et Newton, furent les principaux représentants. Descartes introduit les mathématiques comme «élément structurant» des sciences, les ramenant à un petit nombre de concepts élémentaires, ce à quoi le savoir rationnel grec aspirait déjà.

Dès lors, la science et spécialement la physique, qui connaîtra d'innombrables succès, devient plus autonome et s'éloigne de la philosophie. Mais depuis ce XVIIe siècle dit moderne, le «modèle » galiléen de la science (atomiste, mécaniste et expérimental) s'est profondément transformé. La science n'interprète plus les phénomènes naturels uniquement comme conséquences d'impulsion ou de résistance mécaniques. Elle est devenue moins objectiviste, mettant en question l'indépendance de l'observateur (le sujet). Rappelons que le concept de sujet, qui prend racine dans le De Anima d'Aristote, est clairement défini par Descartes comme le $J e$ du «Je pense, je suis » (Cogito). Le sujet c'est celui (l'ego) qui pense et qui doute et qui est donc supposé du fait même qu'il y a pensée et doute.

$\mathrm{Au}$ XXe siècle, le dialogue entre science et philosophie s'est plutôt renoué. La philosophie tire enseignement de la science pour repenser ses concepts et son ontologie. La science en appelle à la philosophie, surtout à ses concepts éthiques et ontologiques. Toutes deux partagent encore de grandes interrogations. Parmi celles-ci, la question de la réalité et celle de la connaissance de cette sont parmi les plus centrales. Mais chaque discipline traite cette question différemment.

Les physiciens cherchent à caractériser les objets réels, indépendamment des contextes et des sujets qui les expérimentent. Pour garantir son objectivité et son universalité, la physique a dû exclure le sujet des lois qu'elle a élaborées.

Pour les philosophes, en revanche, la relation mutuelle entre réalité (l'objet) et pensée (le sujet) est indissoluble et fait continuellement question. C'est Descartes (1596-1650) qui a structuré le problème de la connaissance d'une manière dualiste en séparant radicalement ces deux pôles :

$\left.1^{\circ}\right)$ le sujet de la connaissance (versant de l'âme, de l'esprit, de la pensée (res cogitans))

$2^{\circ}$ ) l'objet à connaître (versant de la matière, du corps (res extensa))

Kant (1724-1804) va plus tard distinguer 3 grands concepts :

$1^{\circ}$ ) le phénomène : «ce qui apparait » et qui s'atteint par les sens.

$2^{\circ}$ ) le noumène : l'objet construit par la connaissance a priori, intellectuelle ou rationnelle et non sensible.

$3^{\circ}$ ) la « chose en soi » : la chose elle-même, inconnaissable, située au-delà du phénomène.

Pour Hegel (1770-1831) la séparation cartésienne rationnelle entre esprit et matière, est artificielle. Il entendra par Idée, l'identité du sujet et de l'objet comme résultat d'un processus de formation du sujet, de son aptitude à construire sa liberté via la création du concept.

\section{Réalisme/Idéalisme}

Les différentes conceptions du rapport entre pensée et réalité, se répartissent entre idéalismes et réalismes. 


\section{1) L'idéalisme}

L'idéalisme est la position pour laquelle les idées, essentielles aux êtres, ont une valeur plus importante que la réalité. Les philosophes grecs, ont très tôt considéré le raisonnement et les idées comme plus fiables que les sens, pour établir une connaissance vraie. Aussi, l'idéalisme a occupé dans l'histoire de la philosophie une très large place. L'idéalisme absolu ou radical, identifie la chose et l'idée. Pour l'idéalisme modéré la chose conserve une existence plus profonde que son idée.

\section{2) Le réalisme}

Le réalisme se définit en philosophie comme la doctrine qui admet une réalité en soi, indépendante de toutes nos possibilités de la connaître. Esse (être) n'est pas équivalent à percipi (percevoir). Le réalisme fait l'hypothèse, que nous pouvons dire au moins une chose vraie sur cette réalité, mais il affirme de plus que la représentation que nous en avons par l'expérience, peut être construite sans référence à une réalité en soi.

La position naturelle d'une majorité de scientifiques est celle d'un «réalisme objectiviste [1] ». Selon cette position, lorsqu'une chose est vérifiée dans un système relatif, nous pouvons faire l'hypothèse de l'existence de cette chose indépendamment de ce système. L'indépendance du contexte et du sujet sont induites selon des critères statistiques ou des marges d'erreur. Le réalisme mathématique, confère quant à lui, une réalité, plus spécifiquement à ce qui n'est connaissable que par des concepts mathématiques, comme l'espace-temps quadrimensionnel.

\section{REALITE KANTIENNE ET REEL PHYSIQUE}

\section{Kant et la réalité}

\section{1) Inconnaissable en totalité ne signifie pas irréel}

Kant, tient la réalité pour essentiellement inconnaissable. Mais son idéalisme transcendantal reste «modéré [2]». Son but n'est pas de rejeter le réel ou l'idée d'une réalité indépendante (le réel en soi) puisqu'il établit la notion de « chose en soi » comme nécessaire, car autrement dit-il « [...] on arriverait à cette proposition absurde qu'un phénomène (ou apparence) existerait sans que rien n'apparaisse [8] », ou en d'autres termes sans que rien ne soit détecté.

Kant pose la question de l'adéquation entre cette « chose en soi » et la représentation que nous en avons, sans pour autant remettre en cause la certitude que nous avons de la réalité de l'objet pensé. Mais dit-il, il nous est impossible de juger de cette adéquation, puisque nous n'aurons jamais accès qu'à des représentations de la chose et non à la «chose en soi ». Ce qui ne veut bien sûr pas dire que notre représentation soit purement a priori, c'est-à-dire sans lien avec la « chose en soi ».

Notre conviction de la réalité repose sur cette notion de «chose en soi », comme horizon de nos jugements, où convergent les caractères que nous pouvons reconnaître à l'objet. La « chose en soi » est un absolu que nous ne pouvons prétendre déterminer, ni par un savoir théorique toujours incomplet, ni par notre expérience sensible.

\section{2) L'a priori kantien, une notion discutée}

Si la notion de forme a priori de l'entendement (causalité) ou de la sensibilité reste d'actualité, de nombreux philosophes (et spécialement des épistémologues) discutent aujourd'hui l'a priori kantien notamment de l'espace. Il nous semble qu'il faut distinguer ici deux étapes dans la constitution de l'objet. D'un côté, les structures a priori de la sensibilité, innées, internes et indépendantes de l'expérience, qui constituent notre appareil de mesure des perceptions. Et de l'autre, les informations (« le divers pur de la sensation » selon Kant [11]). Non seulement la structure de l'espace en tant que « divers pur », est indépendante de notre appareil de connaissance, mais l'espace se révèle bien plus complexe que ce que notre appréhension nous permet d'en savoir. C'est que confirment les travaux contemporains en psychologie et neurosciences ou en matière d'évolution, qui suggèrent même fortement que notre appareil de perception et d'interprétation serait le fruit d'une lente adaptation à un « espace réel ». Par ailleurs, la notion d'espace envisagée par Kant est déterminée par le niveau de connaissance mathématique de son époque, les géométries non-euclidiennes étaient à peine « inventées ». En bref, ce qu'il nous faut maintenant entendre comme l'espace réel, est de mieux en mieux approché par science.

On peut toutefois entendre l'idéalisme kantien comme une forme de réalisme, du type du « réalisme ouvert » de d'Espagnat, dont la thèse générale est «[...] qu'il y a quelque chose dont l'existence n'est pas tributaire de la pensée » [3], une thèse qui fait l'objet d'une conviction unanime et constitue donc un point de départ minimum satisfaisant.

\section{E. Réalité pratique}

1) Réalité pratique des objets courants 
De tout temps l'être humain a eu besoin de déterminer la réalité des objets. La réalité pratique correspond aux besoins courants de l'être humain. Cette notion de réalité n'est pas absolue comme la « chose en soi » de Kant, mais probabiliste.

Le mécanisme de l'évolution nous a munis d'un système de perception constitué de capteurs et de fonctions psychiques d'analyse (formes a priori chez Kant) qui nous permettent d'établir rapidement la réalité des objets (et des êtres) courants (permanence d'objets, mémorisation des propriétés des objets rencontrés). L'être humain a développé des technologies capables d'étendre nos sens à des objets trop petits ou trop gros, pour observer, évaluer et manipuler une grande partie des objets de notre classe de réalité.

Notre jugement repose par expérience, sur la convergence, la cohérence et l'évolution de représentations multiples de la chose. De plus, l'inconnaissabilité kantienne, ne s'oppose pas à l'évolution du savoir, et au fait que tout inconnaissable puisse devenir connaissable.

Par exemple, Lorsque nous conduisons à grande vitesse des automobiles, la réalité objective des obstacles que nous évitons, nous semble indiscutable et les dangers tout aussi réels.

D'autre part, le degré de réalité pratique d'un objet physique est proportionnel au nombre de paramètres et de propriétés que l'on peut attribuer à cet objet.

L'homme a toujours cherché à classer les objets qu'il rencontrait. Reconnaître la réalité d'un objet physique consiste à le placer dans une classe de réalité déterminée par notre expérience usuelle ou de l'utiliser pour ouvrir une nouvelle classe s'il s'agit d'un objet nouveau. La classe des objets physiques courants correspond à celle des pierres, des rochers, des morceaux de métal ou de matière dure. Une météorite ou une pièce automobile pourra y trouver sa place. Les propriétés communes de ces objets sont :

$1^{\circ}$ ) Ils sont des objets au sens du schème de la permanence d'objet de Piaget.

$2^{\circ}$ ) Ils sont indéformables par manipulation.

$3^{\circ}$ ) Ils n'évoluent pas avec le temps ou très lentement.

$4^{\circ}$ ) Leur poids varie généralement avec leur taille.

$5^{\circ}$ ) Les petits peuvent être lancés à la main.

Cette classification primitive peut être modifiée, étendue, dédoublée pour y inclure de nouvelles propriétés, comme une certaine déformabilité des objets. Toutefois la classe des objets non-déformables par des moyens humains semble se conserver.

On doit dénoncer ici une interprétation abusive, malheureusement courante, de l'idéalisme de Kant, qui assimile l'essentielle inconnaissabilité de la «chose en soi », à une impossibilité absurde, de parvenir à une connaissance raisonnable (réalité pratique) de la réalité du monde qui nous entoure, par nos perceptions, nos sens ou même indirectement par les appareils que nous avons construit.

\section{2) Caractéristiques d'un objet}

L'examen d'un objet par un sujet amène à définir des paramètres évalués qualitativement et quantitativement relativement à un critère de précision. Par exemple la forme de l'objet, (cube, sphère, ...) qui amène à définir des paramètres comme le diamètre, et d'autres paramètres comme la masse, la nature, la résistance à la pression. Cet ensemble de paramètres n'est jamais limité, on peut toujours en rajouter. Ceci est proche de la connaissance absolue de Kant. On peut décomposer l'ensemble des propriétés d'un objet en deux parties : Ses paramètres principaux, en nombre finis (forme, dimensions, poids, matériau,.... associés à leurs précisions respectives) et ses paramètres secondaires (comme l'inhomogénéité de la matière qui le constitue, ses irrégularités par rapport à sa forme idéalisée,,... .).

L'ensemble des paramètres d'un objet possède des critères de cohérence par classe d'objet. De cette liste de nombreux paramètres, on ne retient, la plupart du temps, que la sous-liste des paramètres principaux.

Reconnaître la « réalité pratique » d'un objet revient à ne considérer que ses paramètres principaux. Comme le bon sens nous le suggère, la réalité d'un objet (ou d'une classe d'objets) doit souvent être déterminée très rapidement à partir des seuls paramètres principaux, pour des questions de sécurité (danger, opportunité,...). Cette détermination rapide de la réalité d'un objet peut se faire par différents moyens :

$1^{\circ}$ ) Par la reconnaissance de l'objet ou de sa classe. 
$2^{\circ}$ ) Par la détermination rapide de quelques caractéristiques, pour un objet non mystérieux.

$3^{\circ}$ ) Par le recoupement cohérent d'informations issues de procédés de mesure différents.

\section{3) Le réel de Bitbol}

Comme l'a montré Bitbol [4] la définition de la notion de réalité trouve intérêt à s'appuyer sur un « découpage en tranches » de l'univers qui nous entoure. Le critère de ce découpage concerne les dimensions des objets que nous examinons.

a) Le monde "macroscopique"

Nous appellerons "macroscopique" le monde qui s'étend entre le nanomètre et la taille du système solaire. Ce niveau est le seul directement accessible à nos sens et à nos appareils de mesure.

b) Le monde "microscopique"

Le monde "microscopique" s'étendra en dessous du nanomètre, jusqu'à une limite encore inconnue. La connaissance que nous avons de ce niveau se réduit aux traces qu'il nous laisse au niveau macroscopique. Notre action sur ce niveau passe indirectement et nécessairement par le niveau macroscopique. Mais ce niveau reste une construction hypothétique.

a) L'univers "galactique"

L'univers "galactique" s'étendra au-dessus de la dimension du système solaire. Son caractère borné ou infini ne nous est pas encore connu.

Les frontières entre ces trois domaines semblent étanches. Nous insisterons plus ici, sur les niveaux macroscopiques et microscopiques.

Par exemple, un objet macroscopique ne peut traverser une table qu'en la brisant. Pour le monde microscopique l'objet et la table sont constitués de particules séparées par beaucoup de vide et donc rien n'interdirait leur interpénétration. D'ailleurs, celle-ci est possible pour les objets du microscopique. Par exemple, un faisceau d'électrons pourra traverser une table en bois sans beaucoup de difficultés. Il est surprenant de constater, que vu du niveau macroscopique, les atomes apparaissent comme des petites billes jointives très solides et manipulables au point qu'on puisse les utiliser pour réaliser des dessins [5].

La réalité d'un objet physique sera donc relative au niveau de réalité dans lequel se situe le sujet. On retrouve ici un certain relativisme du point de vue subjectif. Le niveau macroscopique qui a notre prédilection n'est autre que le niveau de l'homme. Et l'on peut imaginer que s'il existait un être intelligent microscopique, ce niveau serait probablement son niveau de référence. La perception qu'il en aurait serait certainement très différente de celle que nous en avons vue de notre niveau macroscopique.

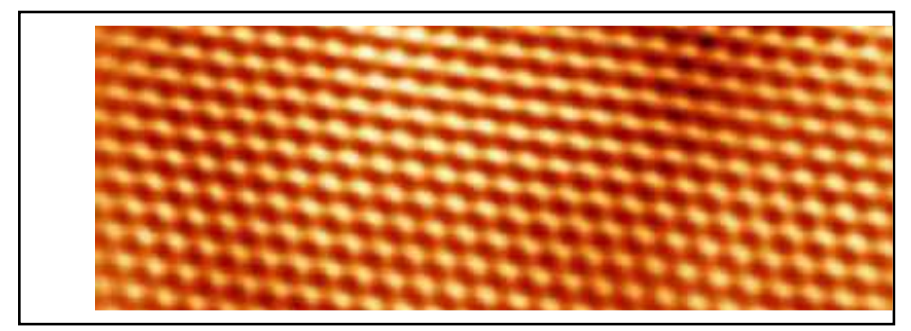

Figure 1. Atomes d'or vus au microscope à effet tunnel.

\section{MODELES ET THEORIES}

\section{B. Notion de Modèle}

La notion de modèle est très générale et dépend des disciplines on l'on se place. Par exemple, les physiciens, définissent la notion de modèle physique comme une simplification ou une schématisation du réel permettant d'en saisir l'essence. Dans le cadre 
de ce travail, nous retiendrons comme définition de la notion du modèle d'un phénomène physique, celle qui est utilisée par les informaticiens et mathématiciens.

Ce type de modèle est un système logico-mathématique, permettant de prédire le comportement d'un objet matériel. Il se compose généralement :

$1^{\circ}$ ) Des données globales qui décrivent l'espace de travail et les effets globaux.

$2^{\circ}$ ) Des données initiales qui décrivent l'état initial du système.

$3^{\circ}$ ) Des variables d'état qui représentent l'état courant du système.

$4^{\circ}$ ) Des expressions mathématiques et logiques qui décrivent l'évolution de l'état de ce modèle.

L'informatique développe de très nombreux modèles destinés à prédire l'évolution des phénomènes (par exemple la météo) ou le comportement d'objets avant de les réaliser (par exemple des avions).

Un modèle sera dit opérationnel s'il décrit précisément les processus physiques qui engendrent l'action. Tandis qu'un modèle non-opérationnel met en œuvre des évaluations qui ne peuvent être obtenues que rétrospectivement, par exemple par un calcul statistique ou d'extremum.

Enfin, nous qualifions un modèle de réel s'il est opérationnel et si, de plus, ses variables et opérations sont prises strictement dans le domaine de réalité considéré [9] ce qui revient à dire qu'un modèle réel décrit ce qui se passe réellement lorsque l'objet est mis en action.

Par exemple : La description de la trajectoire d'une bille roulant sur une surface gauche pourra être qualifiée de réelle si le mouvement de cette bille est décrit par un système d'équations différentielles tenant compte de l'effet de la pesanteur, de la réaction de la surface et de l'inertie sur la bille en utilisant uniquement des opérations réalisables directement dans le niveau de réalité macroscopique. Par contre, la description de cette trajectoire par une relation d'optimalité comme le principe de Maupertuis met en œuvre des mécanismes qui ne sont manifestement pas ceux à l'œuvre pour produire les mouvements de la bille.

L'expérience montre que la précision d'une prédiction peut être indépendante du critère de réalité de son calcul. Des modèles parfaitement non-réels peuvent fournir des prédictions particulièrement justes.

Les critères de non-réalité (statistiques ou d'optimalité) découlent souvent de l'analyse, à posteriori, du comportement de l'objet. Ce mode de calcul ne peut, en aucun cas, décrire l'origine du mouvement, donc l'opérationnalité du phénomène. Vouloir leur donner un sens réel supposerait que l'on dispose d'un moyen d'analyse préalable des caractéristiques de toute la zone d'évolution du phénomène, d'un dispositif de calcul capable de déterminer la trajectoire optimale et d'un dispositif de télécommande capable de piloter la bille à partir de ces calculs.

Les modèles réels peuvent permettre de découvrir de nouveaux phénomènes physiques, par l'analyse inductive du sens des nécessités mathématiques posées pour le bon fonctionnement du modèle par rapport au monde réel.

Par exemple, la découverte de la circulation aérodynamique autour d'une aile d'avion par Nikolaï Joukovski a découlé de la nécessité d'introduire un flux d'air tournant autour d'une section d'aile transformée en un cylindre par la transformation de Joukovski. Par contre, l'application du même processus sur des modèles non-réels, peut conduire à des raisonnements erronés en suggérant une fausse réalité.

\section{Notion de théorie}

Une théorie, plus générale qu'un modèle, est un objet cognitif qui prétend décrire et expliquer la nature et le comportement réel d'un phénomène. Elle peut occuper plusieurs degrés de certitude : Elle commence généralement par être "proposée", c'est-à-dire induite à partir de données antérieures. Elle devient ensuite "validée" lorsqu'elle est confirmée par des résultats expérimentaux. La démonstration de sa fausseté, signe généralement son arrêt de mort. Une théorie est donc nécessairement un modèle réel, ou supposé comme tel.

\section{1) La réalité des théories}

Le caractère spéculatif des théories proposées peut excuser un caractère temporaire d'irréalité, maintenu quelquefois plus que nécessaire, malgré l'apparition de théories plus réelles (par exemple pour la théorie de la décomposition de Fourrier maintenue au niveau d'une théorie malgré le développement de celle des ondelettes). 


\section{a) Un exemple de théorie non-réelle}

Un exemple nous montrera comment une théorie peut s'avérer non-réelle.

Considérons la décomposition d'un bip sonore unique, émis dans une chambre sourde, en ondes sinusoïdales par l'intégrale de Laplace. Un ensemble de microphones reliés à un analyseur de spectre affiche le spectre continu de ce signal peu après son émission. La théorie de ce genre de décomposition stipule que les ondes sinusoïdales composant ce spectre, s'étendent sur un intervalle temporel allant de $-\infty$ à $+\infty$. Avant et après le signal initial, la composition de ces signaux produit un signal nul. La recomposition partielle d'une sélection de quelques-unes de ces composantes, en respectant leurs amplitudes et leurs phases respectives, fournit une impulsion voisine du signal initial.

Nous pouvons déjà noter que ce raisonnement contient une (énorme!) faille de causalité, dans le sens où ces composantes sont émises, avec une phase et une intensité convenables pour participer à la construction du bip, bien avant l'émission de celui-ci !

Puisque ces composantes sont censées exister, avec un niveau et une phase constants, et ceci depuis l'aube des temps, il devrait être possible de les détecter avant l'occurrence du bip, grâce à des filtres suffisamment étroits. L'analyse de la phase de quelques composantes devrait alors permettre de calculer la date à laquelle ce bip sera émis. Un tel raisonnement vient tout juste de nous permettre de construire une machine à prévoir le futur, ce qui s'est toujours révélé impossible, sauf pour quelques auteurs de science-fiction.

L'erreur, car il y en a une, se situe dans le choix de la nature des composantes dont l'existence s'étend de $-\infty$ à $+\infty$. Pourtant, malgré cette importante faille de causalité, cette décomposition spectrale est utilisée journellement pour calculer des filtres et autres organes utilisés pour le traitement du signal. Son application donne des résultats très précis qui permettent de calculer des appareils électroniques remplissant parfaitement leurs fonctions.

Pour ces applications pratiques, les ingénieurs savent par expérience qu'il ne faut pas utiliser les composantes d'une décomposition hors des limites de définition du signal.

Pour contrer ce qu'il convient d'appeler un bricolage, les mathématiciens ont inventé la théorie de la décomposition en ondelettes [12] qui utilise des signaux de base de la décomposition dont la définition reste dans la limite de celle du signal initial.

\section{2) Rapport entre théories}

\section{a) Hiérarchie entre théories}

Il nous est apparu que les théories physiques sont la plupart du temps, organisées de manière hiérarchique, comportant à leur base des modèles réels surmontés de modèles non-réels.

Dans l'exemple de la bille roulant sur une surface gauche, le modèle réel s'appuyant sur la mécanique du roulement de la bille peut être considéré comme constituant un premier niveau de modélisation du phénomène. Un second niveau peut être constitué par le modèle non-réel utilisant le principe de moindre action de Maupertuis, ou les équations de Lagrange ou d'Hamilton qui en découlent. Dans cette hiérarchie, chaque niveau gagne en concision et élégance, mais perd en réalité.

\section{b) Théories concurrentes}

Dans quelques cas très particuliers, plusieurs théories différentes peuvent prétendre expliquer le même phénomène avec des degrés comparables de réalité.

A titre d'exemple, nous pouvons citer les théories de la gravitation de Newton dans laquelle la force de gravité est une propriété de la matière elle-même. Einstein propose, dans le cadre de la relativité générale, l'idée que la gravité serait la force inertielle dans un espace - l'éther ! - déformé par la présence des masses elles-mêmes. Une troisième théorie de la gravitation est basée sur un effet d'entraînement des masses par des particules et leur masquage par ces mêmes masses. Emise initialement par Nicolas Fatio de Duillier en 1690, puis reprise et développée par Georges-Louis Lessage en 1798, cette théorie fut finalement formalisée et a récemment mené à une expérience d'antigravité par Claude Poher [4]. La relativité générale semble avoir la faveur des milieux scientifiques, mais la compétition avec la théorie de Poher-Lessage est loin d'être tranchée.

La mécanique quantique a été développée, vers 1930, en deux versions par Heisenberg d'une part (comme mécanique matricielle) et par Schrödinger de l'autre (comme mécanique ondulatoire). Ces deux théories formellement très différentes, l'une interprétable en termes discrets l'autre en processus continus, prédisent les mêmes faits observables. La mécanique quantique dite «standard » et la théorie quantique de Böhm, font exactement les mêmes prédictions empiriques mais reposent sur des descriptions 
du monde physique, incompatibles (l'une indéterministe l'autre déterministe). Mais la théorie la plus récente prédit «outre les mêmes évènements observables que l'ancienne, des évènements sur lesquels l'ancienne théorie ne se prononcent pas [13]».

\section{c) Sous-détermination des théories}

D’une manière générale les théories sont souvent sous-déterminées par l'expérience. Les stimuli sensoriels n'imposent pas l'unicité d'un système d'énoncé, mais restent compatibles avec plusieurs de ces systèmes.

\section{3) Non-réalité de la mécanique quantique}

La mécanique quantique a été imaginée entre 1913 et 1930 par un groupe de physiciens de premier plan, pour rendre compte de nouveaux phénomènes physiques, comme la photo-électricité, observés à la charnière des XIXème et XXème siècles. Les objets suspectés d'être responsables de ces effets étant manifestement trop petits pour être observés, ces nouveaux concepts restèrent purement fictifs. D'autre part, leur conception s'est inspirée des principes physiques les plus avancés de l'époque (Maupertuis, Lagrange, Hamilton), mais aussi les plus non-réels. Tous les savants qui ont participé à ce grand œuvre savaient que ces concepts ne correspondaient certainement pas à la réalité des phénomènes, mais seulement à leur comportement moyen. Le "tais-toi et calcule" de Feynman en témoigne. Toutefois la surprenante applicabilité et la précision des résultats, amena de nouvelles générations de physiciens à assimiler ce modèle à une réalité, de manière dogmatique. Si bien qu'il est aujourd'hui devenu très difficile de remettre en cause ce dogme et de rappeler qu'il ne s'agissait à l'origine que d'un modèle.

La mécanique quantique entretient une certaine obscurité et un certain hermétisme. Elle a été conçue dans l'idée que ses objets resteraient définitivement inaccessibles. Or l'évolution de la technologie nous a permis d'atteindre ce qui était autrefois inimaginable. Il est maintenant possible de voir les atomes et de les manipuler avec des microscopes à effet tunnel et plus récemment, d'apercevoir l'orbitale d'atomes d'hydrogène.

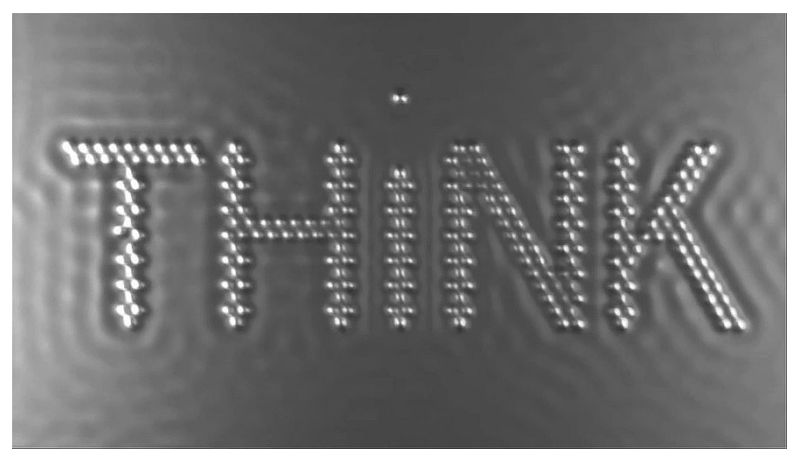

Figure 2. Think écrit avec des molécules de $\mathrm{CO}$ positionnées sur une plaque de cuivre. à $-268,15^{\circ} \mathrm{C}$ par un microscope à effet tunnel. Chaque image fait 45 x $25 \mathrm{~nm}$. Extrait du film : A Boy and His Atom (IBM Research - Almaden)

\section{CONCLUSION}

Nous avons tenté de montrer dans cet exposé que, moyennant certaines définitions pragmatiques, on peut définir une notion de «réalité pratique» propre à la physique, conforme à l'usage et si elle n'est pas exclusive, compatible avec la plupart des conceptions philosophiques. Nous avons notamment montré que la critique kantienne ne s'oppose ni à l'adoption de notre notion de « réalité pratique », ni à une démarche scientifique qui s'en prévaudrait.

En résumé, nous avons retenu deux formes d'excès, tous deux indéfendables :

$1^{\circ}$ ) La position de l'idéalisme radical, selon laquelle l'objet physique n'est relatif qu'à l'esprit :

Cette position ne tient pas, car notre conviction de la réalité repose sur la supposition nécessaire d'une «chose en soi » comme concept limite, un horizon où convergent les propriétés que nous reconnaissons à l'objet. Croire en la réalité d'un 
objet, c'est admettre que ce n'est pas notre esprit qui est la source des phénomènes qui le fondent, mais une chose extérieure à l'esprit, qui n'est pas constituée par l'activité psychique du sujet.

$2^{\circ}$ ) La démarche qui consiste à déduire de sa seule exactitude prédictive, le caractère de réalité d'un modèle ou une théorie, seulement établi par construction :

Cette démarche nous l'avons vu, présente des limites évidentes, et peut vite s'avérer incompatible avec «l'attitude naturelle », à savoir le critère d'une certaine évidence ontologique, qui reste en dernier lieu la position la plus empreinte de sincérité. C'est la raison pour laquelle les critiques régulièrement adressées au sensible sont rarement probantes. Pour attribuer une certaine réalité à un modèle ou une théorie, son opérationnalité reste certes, une exigence difficilement contournable. Mais il est clair qu'une confirmation sensible au niveau macroscopique renforce, voire scelle la profonde conviction que nous avons de sa réalité.

Pour ces raisons il nous semble qu'il est utile et même nécessaire de repréciser la notion de «réalité pratique » dans le champ des sciences et spécialement de la physique, travail que nous avons amorcé ici.

\section{REFERENCES}

[1] B. d'Espagnat, Traité de physique et de philosophie, Paris, Fayard, 2002, p. 36.

[2] E. Kant, Critique de la raison pure, trad. de F. Alquié, Paris, Gallimard, 1980, Antinomie des raisonnements dialectiques, Sixième section.

[3] B. d'Espagnat, Traité de physique et de philosophie, Paris, Fayard, 2002, p. 35.

[4] M. Bitbol, La nature est-elle un puits sans fond, La Recherche ${ }^{\circ} 405$, fev 2007, p. 31

[5] A Boy and His Atom, film IBM Research, mai 2013.

[6] A.S. Stodolna and all, Hydrogen Atoms Under Magnification : Direct Observation of the Nodal Structure of Stark States, Physical Review Letters, PRL 110, 213001, 5 pages, 24 may 2013

[7] C. Poher, D. Poher, P. Marquet, Propelling phenomenon revealed by electric discharges into layered Y123 superconducting ceramics, The European physical Journal Applied Physics 50, 30803 (2010), 11 pages.

[8] E. Kant, Critique de la raison pure, trad. de F. Alquié, Paris, Gallimard, 1980, Préface de la seconde édition.

[9] M. Bitbol, La nature s'organise comme les poupées russes, in Les Dossiers de La Recherche, N. 43, mai 2011.

[10] La recherche ${ }^{\circ} 489$ juillet 2014. La réalité n'existe pas.

[11] M. Bitbol \& S. Laugier, Physique et réalité (Un débat avec B. d'Espagnat), Paris, Editions Frontières-Diderot, 1997

[12] Y. Meyer, Ondelettes et applications, Masson, Paris, 1993 ; Ondelettes et opérateurs, Herman, Paris, 1989

[13] L. Soler, Introduction à l'Epistémologie, Paris, Ellipses, 2009. 
Acta Europeana Systemica $\mathrm{n}^{\circ} 4$ 